\title{
Long term follow-up of surgery management of prostate leiomyosarcoma metastasized to the rib: A case report and literature review
}

\author{
WEI CHEN, DENG-JUN HAN, GUANG-QING FU, WEI LIN and YONG LIANG
}

Department of Urology, Zigong No. 4 People's Hospital, Zigong, Sichuan 643000, P.R. China

Received January 8, 2016; Accepted April 1, 2016

DOI: $10.3892 / \mathrm{mco} .2016 .898$

\begin{abstract}
Prostate sarcoma, particularly the pathological type of leiomyosarcoma, is a rare carcinoma, which originated from the interstitial tissue of the prostate. This sarcoma type has a poor prognosis. This disease accounts for $\sim 0.1 \%$ of all prostate cancer and it usually occurrs in patients aged between 40 and 78-years-old. Although prostate leiomyosarcoma has a poor prognosis, early treatment of post-operative recurrence and metastases via a whole-body examination and closer follow-up was possible. These measurements may significantly prolong the survival time and improve the quality of life. The present study reported a successful case of surgical management for prostate leiomyosarcoma in the Zigong No. 4 People's Hospital (Sichuan, China) during 1995 until 2015, with post-operative follow-up for 20 years.
\end{abstract}

\section{Introduction}

Prostate sarcoma is a rare type of carcinoma, which originated from the interstitial tissue of the prostate. The predominant symptom is frequent urination, dysuria and hematuria. Its etiology remains unknown. The common pathological classification involved rhabdomyosarcoma, leiomyosarcoma, and fibrosarcoma. This disease normally occurs in middle-aged patients. Prostate sarcoma always has a poor prognosis and it accounts for $\sim 0.1 \%$ of all prostate cancer cases (1). It is reported that a quarter of patients already exhibit a metastatic mass at the time of diagnosis. Markowski et al (2) reported that the median overall survival time of prostate sarcoma is 9 months in patients with bladder invasion and 7.1 months in patients with metastatic disease. The present study reported a successful case of surgical management for prostate leiomyosarcoma during 1995 until 2015, with post-operative follow-up

Correspondence to: Dr Yong Liang, Department of Urology, Zigong No. 4 People's Hospital, 2 Tanmulin Street, Zigong, Sichuan 643000, P.R. China

E-mail: cweimed@163.com

Key words: prostate, sarcoma, leiomyosarcoma, immunohistochemistry, prognosis for 20 years. The present study also aimed to perform a literature review, in order to correctly diagnose patients, and thus establish an effective treatment plan both individually and collectively.

\section{Case report}

A 58-year-old male presented with frequent micturition and dysuria since 1995, which went without any treatment until the persistent hematuria appearance for 4 days prior to hospitalization. The symptoms were becoming progressively worse the past few days. The patient was a smoker and drank alcohol socially. No genitourinary cancer history existed in his family. Rectal examination revealed a significant nodule around the area of the prostate, $\sim 4-5 \mathrm{~cm}$ in diameter. The laboratory examination revealed that at presentation, the acid phosphatase (ACP) was $1.6 \mathrm{U} / 1$ and prostate specific antigen (PSA) was $4.6 \mu \mathrm{g} / 1$. The ultrasound guided transrectal prostatic biopsy confirmed the histological type was prostate sarcoma (Fig. 1A). As a result, the patient received the radical prostatectomy in April 1995. Following the surgery, the hematuria and dysuria disappeared. The post-operative pathological diagnosis was prostate leiomyosarcoma (Fig. 1B). At the 2 year follow-up, the hematuria was observed again, and increased pain was exhibited at the hypogastrium. A B-ultrasound examination of the abdomen revealed a slight nodule $(\sim 1.8 \mathrm{~cm}$ in diameter) in the centre of the prostate (Fig. 1C). A chest $\mathrm{x}$-ray and emission computed tomography (ECT) examination revealed no metastatic masses. The present study considered this as recurrent prostate sarcoma, and as a result, radical prostatectomy, radical cystectomy and reconstruction of the bladder with ileum were performed. After 1 year, the patient found a hard mass $(\sim 3.3 \mathrm{~cm}$ in diameter) on the right side of the back, without any clinical symptoms. A chest x-ray and B-ultrasound revealed that the mass as a metastatic carcinoma from the prostate. An ECT whole-body bone scan revealed a striking concentration of radioactivity in the right ninth rib. The patient received partial rib resection of the right ninth rib. The post-operative pathological diagnosis was leiomyosarcoma of the right chest wall (Fig. 1D and E). At the 10 month follow-up, a new mass was revealed at the operative site. The patient had to receive a secondary operation of the right ninth rib. Immunohistochemical results revealed the following: $\alpha \mathrm{AT}$ $(-)$, Actin (+), Vimentin ( \pm ), CK (-) and S100 (++) (Fig. 1F). 

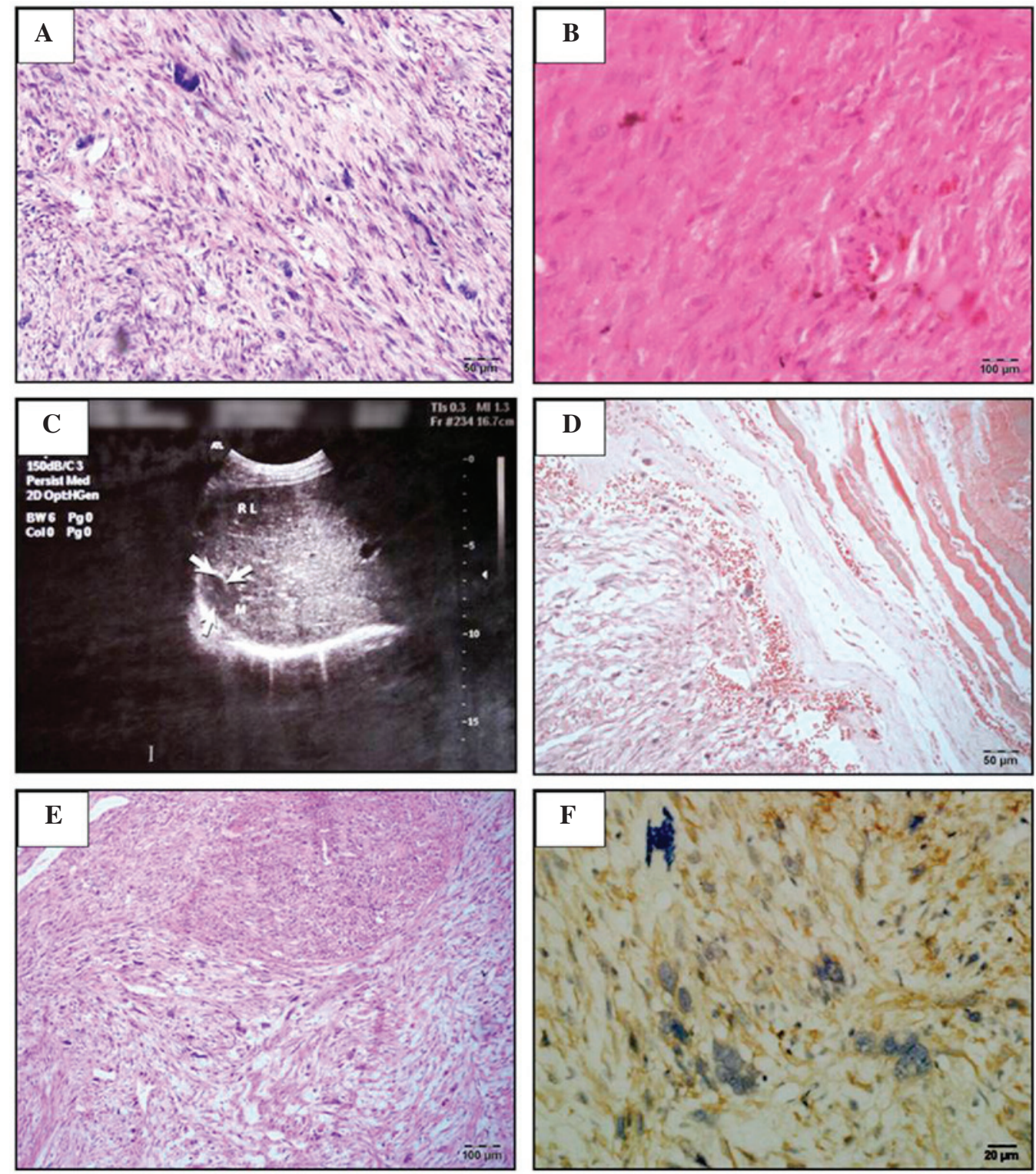

Figure 1. Assessment of the biopsy samples at various stages. (A) The first prostate biopsy slice, stained with H\&E, revealed tumor cells were predominantly spindle-shaped, the cytoplasm was dyed in red, nuclei were rod-shaped and rounded at each end. The majority of the tumor cells were with mild to moderate atypia and occasionally giant tumor cells were visible (magnification, x20). (B) A biopsy slice of the first recurrence tumor, stained with H\&E, revealed that tumor cells were in long spindle-shaped or oval and arranged in a palisade. Significant cellular atypia was observed and the cytoplasm was dyed in bright red. Mitotic cells were easily detected and a section of the tumor cell exhibited large nuclei, and interstitial was more loose (magnification, x20). (C) The right bib metastatic cancer observed by the abdominal B-ultrasound results (a solid mass $\sim 2.2 \times 1.7 \mathrm{~cm}$ ) in the capsule between diaphragm and liver. (D) A biopsy slice of the first resection of the rib metastases, stained with Masson-Goldnen trichrome, revealed red tumor cells were red (magnification, x20). (E) A biopsy slice of the first resection of the rib metastases, stained with H\&E (magnification, x10). (F) A biopsy slice of the second resection of rib metastases, stained with actin antibody (magnification, $\mathrm{x} 40$ ).

To date, with 17 years of follow-ups, the patient has exhibited no recurrence.

\section{Discussion and literature review}

Sarcoma of the prostate is a rare malignant carcinoma of genitourinary cancer. According to different histological types, it can be divided into prostate leiomyosarcoma, rhabdomyosarcoma, fibrosarcoma and spindle cell sarcoma (3). The anatomic structure of prostate leiomyosarcoma was the mesenchymal tissue in the prostate smooth muscle. The development is rapid and as early as the time to reveal a metastatic mass. This disease accounts for $\sim 0.1 \%$ of all prostate cancer cases and it normally occurs in patients aged between 40 and 78 -year-old (1). Clinical symptoms are not normally significant. The majority of the symptoms revealed only mild to moderate urinary tract obstructive symptoms, including frequent urination, urine urgency, voiding difficulty, hematuria and perineal pain. No significant abnormal changes were observed in the prostatic physical examination, and the prostate volume and serum PSA levels remain in the normal range (4), whereas the imaging examination, including B-type ultrasound can often be reveal the early stage carcinoma as it always develops with an invasive growth.

The differential diagnosis of prostate leiomyosarcoma includes leiomyosarcoma, which originated in the bladder or 
adjacent to the predominant blood vessels, as well as other rare benign or malignant primary prostate spindle cell tumors. Imaging or radiology examination, including computed tomography, may be important in the identification. The most common confirmed diagnostic method was the B-ultrasound guided transrectal prostate biopsy or transurethral resection of the prostate. Additionally, Barwad et al (5) reported that fine needle aspiration cytology can also be adopted for the initial diagnosis of prostate leiomyosarcoma. Prostate leiomyosarcoma tissue exhibited moderate to severe abnormal spindle cells cross-enriched under the microscope. The degree of cell atypia, the lesion site, the degree of mitosis and the rate of infiltration can be used to distinguish the low-grade malignant smooth muscle sarcoma and leiomyoma on the pathological section. A closer association was observed between the degree of atypia/mitosis and the characteristics of the disease. Even though it was reported that leiomyosarcoma cells with more atypia or mitosis tend to have higher degree of malignancy (6), certain low-grade prostate leiomyosarcoma were reported (7). To differentiate from stromal sarcoma, leiomyosarcoma lacks normal glands, in addition to the coated glands.

Radical prostate resection was the major measurement on the treatment of prostate leiomyosarcoma. The extent of surgical resection markedly affected the prognosis. An improved prognosis occurs in the microscopic negative margin compared with the positive ones. To date, no optimal treatment of this disease exists. Scholars have suggested that pre-operative adjuvant therapy, followed by radical surgery or post-operative adjuvant therapy, may provide an improved prognosis (8). Radical surgery can often be defined as both radical prostate and bladder resection, and it always involves pelvic lymph node dissection. Current treatment opinions for locally advanced prostate leiomyosarcoma are neoadjuvant chemotherapy or neoadjuvant radiochemotherapy, where chemotherapy may involve ifosfamide, cyclophosphamide or dacarbazine (9). Other literature also reported that chemotheraphy with methotrexate + etoposide + cisplatin may benefit a similar outcome (10). However, no consensus on the radiation dose exists. Sakano et al (11) suggested that local control can be achieved when the radiation dose was controlled between $\sim 60$ and $66 \mathrm{~Gy}$. Since the disease is more prone to local and distant recurrence, physical and other auxiliary examination, including chest and abdomen x-ray and whole-body bone scan for patients readmitted is highly important. Another important factor, to make a comprehensive examination, was the regular follow-up of a patient, which is difficult under the current constrained clinical situation caused by various factors.

According to the literature, the prognosis of patients with prostate leiomyosarcoma is poor. It is reported that $\sim 25 \%$ of patients already exhibit a metastatic mass at the time of diagnosis, and the predictive survival interval was 2-5 years following diagnosis in 50-75\% of patients. The lung metastases can be found on the other patients in a short period of time (12). Vandoros et al (13) performed a comprehensive analysis based on 54 prostate leiomyosarcoma cases in the published literature prior to 2008 . In this previous study, $23.5 \%$ of patients exhibited metastatic disease at the time of diagnosis. The proportion of lung metastasis was $\sim 17.6 \%$, followed by $\sim 11.7 \%$ of liver metastases, $5.8 \%$ of bone metastases and only $3.6 \%$ of brain metastases.

The present case was without obvious metastases at the time of diagnosis, with early post-operative recurrence and distant metastasis. Therefore, although prostate leiomyosarcoma has a poor prognosis, early treatment of post-operative recurrence and metastases via a whole-body examination and closer follow-up was realistic. These measurements may significantly prolong the survival time and improve the quality of life for patients.

\section{Acknowledgements}

The present study was funded by grants from The Key Project of Science and Technology of Zigong (no. 2015SF03) and the Scientific research project of Health Department of Sichuan Province (no. 110590).

\section{References}

1. Hansel DE, Herawi M, Montgomery E and Epstein JI: Spindle cell lesions of the adult prostate. Mod Pathol 20: 148-158, 2007.

2. Markowski MC, Eisenberger MA, Zahurak M, Epstein JI and Paller CJ: Sarcomatoid carcinoma of the prostate: retrospective review of a case series from the Johns Hopkins Hospital. Urology 86: 539-543, 2015.

3. Janet NL, May AW and Akins RS: Sarcoma of the prostate: A single institutional review. Am J Clin Oncol 32: 27-29, 2009.

4. Talapatra K, Nemade B, Bhutani R, Kane S, Bakshi A, Muckaden MA and Laskar S: Recurrent episodes of hematuria: A rare presentation of leiomyosarcoma of prostate. J Cancer Res Ther 2: 212-214, 2006.

5. Barwad A, Khandelwal N, Vyas S, Gogoi D and Dey P: Primary leiomyosarcoma of the prostate with lung metastasis: Report of a case diagnosed by fine-needle aspiration cytology. Diagn Cytopathol 39: 700-702, 2011.

6. Tavora F, Kryvenko ON and Epstein JI: Mesenchymal tumours of the bladder and prostate: An update. Pathology 45: 104-115, 2013.

7. Dominguez A, Piulats JM, Suárez JF, Condom E, Castells M, Camps N, García Del Muro FX and Franco E: Prostatic sarcoma after conservative treatment with brachytherapy for low-risk prostate cancer. Acta Oncol 52: 1215-1216, 2013.

8. Hayashi T, Nakai Y, Kakuta Y, Takayama H, Nakayama M, Nonomura N, Okumi M and Nakao A: A case of leiomyosarcoma of prostate: Multimodality therapy suppressed disease progression for long term. Hinyokika Kiyo 56: $527-530,2010$

9. Herawi M and Epstein JI: Specialized stromal tumors of the prostate: A clinicopathologic study of 50 cases. Am J Surg Pathol 30: 694-704, 2006.

10. Suppiah R, Wood L, Elson P and Budd G: Phase I/II study of docetaxel, ifosfamide, and doxorubicin in advanced, recurrent, or metastatic soft tissue sarcoma (STS). Invest New Drugs 24: 509-514, 2006.

11. Sakano Y, Yonese J, Okubo Y, Yoshimura K, Maeda H, Yamauchi T, Fukui I, Kawai T and Ishikawa Y: Leiomyosarcoma of the prostate: A case report of remission for 9 years by radiotherapy. Hinyokika Kiyo 41: 629-632, 1995 (In Japanese).

12. Martini N and McCormack PM: Evolution of the surgical management of pulmonary metastases. Chest Surg Clin N Am 8: 13-27, 1998.

13. Vandoros GP, Manolidis T, Karamouzis MV, Gkermpesi M, Lambropoulou M, Papatsoris AG, Zachos I and Konstantinopoulos PA: Leiomyosarcoma of the prostate: Case report and review of 54 previously published cases. Sarcoma 2008: 458709, 2008. 